\title{
Molecular epidemiology of residual Plasmodium vivax transmission in a paediatric cohort in Solomon Islands
}

\author{
Yi Wan Quah ${ }^{1,2}$, Andreea Waltmann ${ }^{1,2}$, Stephan Karl ${ }^{1,2}$, Michael T. White ${ }^{3}$, Ventis Vahi ${ }^{4}$, Andrew Darcy ${ }^{4}$, \\ Freda Pitakaka ${ }^{4}$, Maxine Whittaker ${ }^{5}$, Daniel J. Tisch ${ }^{6}$, Alyssa Barry ${ }^{1,2}$, Celine Barnadas ${ }^{1,2}$, James Kazura ${ }^{6}$ \\ and Ivo Mueller ${ }^{1,2,3^{*}}$ (D)
}

\begin{abstract}
Background: Following the scale-up of intervention efforts, malaria burden has decreased dramatically in Solomon Islands (SI). Submicroscopic and asymptomatic Plasmodium vivax infections are now the major challenge for malaria elimination in this country. Since children have higher risk of contracting malaria, this study investigated the dynamics of Plasmodium spp. infections among children including the associated risk factors of residual P. vivax burden.

Methods: An observational cohort study was conducted among 860 children aged 0.5-12 years in Ngella (Central Islands Province, SI). Children were monitored by active and passive surveillances for Plasmodium spp. infections and illness. Parasites were detected by quantitative real-time PCR (qPCR) and genotyped. Comprehensive statistical analyses of $P$. vivax infection prevalence, molecular force of blood stage infection ( ${ }_{\text {mol }} \mathrm{FOB}$ ) and infection density were conducted.

Results: Plasmodium vivax infections were common (overall prevalence: 11.9\%), whereas Plasmodium falciparum infections were rare (0.3\%) but persistent. Although children acquire an average of 1.1 genetically distinct $P$. vivax blood-stage infections per year, there was significant geographic heterogeneity in the risks of $P$. vivax infections across Ngella (prevalence: 1.2-47.4\%, $p<0.01$; molFOB: 0.05-4.6/year, $p<0.01$ ). Malaria incidence was low (IR: 0.05 episodes/ year-at-risk). Age and measures of high exposure were the key risk factors for $P$. vivax infections and disease. Malaria incidence and infection density decreased with age, indicating significant acquisition of immunity. G6PD deficient children (10.8\%) that did not receive primaquine treatment had a significantly higher prevalence ${ }_{a} \mathrm{OR}: 1.77, \mathrm{p}=0.01$ ) and increased risk of acquiring new bloodstage infections ( mol $F O B$ IRR: 1.51, $p=0.03$ ), underscoring the importance of anti-relapse treatment.

Conclusion: Residual malaria transmission in Ngella exhibits strong heterogeneity and is characterized by a high proportion of submicroscopic and asymptomatic $P$. vivax infections, alongside sporadic $P$. falciparum infections. Implementing an appropriate primaquine treatment policy to prevent $P$. vivax relapses and specific targeting of control interventions to high risk areas will be required to accelerate ongoing control and elimination activities.
\end{abstract}

Keywords: Plasmodium vivax, Solomon Islands, Asymptomatic, Cohort, Heterogeneity

\footnotetext{
${ }^{*}$ Correspondence: mueller@wehi.edu.au

1 Population Health and Immunity Division, The Walter and Eliza Hall

Institute of Medical Research, Parkville, VIC, Australia

Full list of author information is available at the end of the article
} 


\section{Background}

Over recent years, collaborative efforts among ministries of health in malaria-endemic countries, international communities and global funders have significantly decreased the transmission and clinical burden of malaria worldwide [1]. The decrease in transmission has resulted in Plasmodium spp. infections becoming more heterogeneous within countries, between regions, among villages and even within households [2-4]. The decline of clinical malaria has often been linked with an increase in low density and afebrile Plasmodium spp. infections [5-7]. These low-density infections are particularly problematic for malaria control and elimination programmes as they are not detected by routine surveillance and constitute a silent reservoir of infections $[8,9]$. Asymptomatic infections may be chronic and untreated Plasmodium falciparum infections can persist for 6 months or more [10]. Meanwhile, asymptomatic Plasmodium vivax relapses may account for $80 \%$ of all $P$. vivax blood-stage infections [11].

In many malaria-affected countries outside sub-Saharan Africa, where both $P$. falciparum and $P$. vivax coexist, a shift in species predominance from $P$. falciparum to $P$. vivax has been observed $[6,12]$. Given its strong preference to invade reticulocytes [13] and the more rapid acquisition of clinical immunity to $P$. vivax [14], low-density, asymptomatic infections tend to constitute a higher proportion of $P$. vivax infections as compared to $P$. falciparum infections. Conventional malaria surveillance approaches focused on monitoring clinical cases [15-18] are thus poorly suited to identify and delineate pockets of residual $P$. vivax transmission [19].

Following substantial progress made in malaria control, the Solomon Islands are aiming for malaria elimination by 2030 [20]. National statistics reported a tenfold decrease in malaria incidence over the last two decades from $442 / 1000$ population in 1992 to $40.5 / 1000$ population in 2015 [21]. In addition, Plasmodium spp. transmission is highly heterogeneous across provinces [22, 23], ranging from high transmission in Makira Province (80.6/1000 population in 2013) to low transmission in Isabel (5.8/1000 population in 2013), and no transmission in Rennell and Bellona Province [21]. Though impressive, these reductions in malaria burden may be fragile and in the context of reduced funding for malaria control and inadequate access to services reported malaria cases escalated to $81.0 / 1000$ in 2016 [24, 25]. A national household survey in 2015 reported that while $86 \%$ of households in the SI own at least one long-lasting insecticide-treated net (LLIN), LLIN usage was reported to only be $57 \%$ [21]. In addition, the prolonged use of insecticides and LLINs in Solomon Islands has resulted in a tendency for Anopheline mosquitoes to bite more frequently outdoors during the early evening instead of biting indoors and later at night [26]. A more in-depth understanding of the nature and risk factors for residual malaria transmission is thus essential for improving the effectiveness of Solomon Islands' malaria control and elimination programmes.

In the two provinces where pilot programmes for malaria elimination were conducted (Isabel and Temotu), an increase in the proportion of malaria caused by $P$. vivax infection were observed as the overall malaria incidence decreased. Furthermore, a higher proportion of low-density and asymptomatic infections, as well as increased spatial clustering of residual malaria transmission risk were observed [22, 27-29]. Less is known about how ongoing control has changed malaria epidemiology in the higher transmission areas of central Solomon Islands. National statistics (based on light microscopic diagnosis) in 2012 reported that $63 \%$ of malaria cases in Central Islands Province were caused by $P$. falciparum while the remaining $37 \%$ of malaria cases were due to $P$. vivax infections $[21,30,31]$. However, $P$. vivax prevalence by qPCR was considerably higher than that of $P$. falciparum ( $13 \%$ vs. $0.14 \%$, respectively) in a cross-sectional survey of 3501 local residents in Ngella, Central Islands Province [23]. Findings from this survey also identified high rates of submicroscopic and asymptomatic Plasmodium spp. infections, and a higher risk of infections among children as compared to adults.

Since cross-sectional surveys only measure Plasmodium spp. infection at a single time point, it is difficult to understand how risk factors identified in the cross-sectional survey may be related to the seasonal patterns of Plasmodium spp. infections in Ngella. From a single-time point study, it is also not possible to determine incidence of Plasmodium spp. infection and illness. Therefore, an in-depth investigation on the burden of asymptomatic and submicroscopic Plasmodium spp. infections among children (6 months to 12 years of age) in Ngella was conducted as part of an 11-month longitudinal, observational cohort study. In order to describe the longitudinal dynamics of Plasmodium spp. infections and disease, 860 children from villages located in North Coast and Bay regions (previously identified with higher Plasmodium spp. transmission rate [23]) were followed and the incidence of $P$. vivax infections and its associating risk factors were studied.

\section{Methods \\ Study site}

This study was conducted in Ngella, Central Islands Province of Solomon Islands. Ngella is located between Malaita (approximately 50 miles north east) and Guadalcanal provinces (approximately 27 miles south and hosts 
the capital city, Honiara). These provinces are connected to Ngella by ferry service and private boats. Their malaria incidences were shown to be moderate to high in 2013 (Honiara: 69.9/1000 population; Guadalcanal: 64.0/1000 population; Malaita: 35.9/1000 population; Central Islands: $55.5 / 1000$ population) [21, 31]. Further details of the study site are described elsewhere [23]. A total of 20 villages in Ngella were selected for this study (Fig. 1) based on: (i) higher prevalence of $P$. vivax infections as noted previously among 10 villages in Ngella [23], (ii) consultation with Ministry of Health officials, (iii) easy accessibility during high tides and stormy weather throughout the year, as well as (iv) number of children in each village.

\section{Field study design}

The study was conducted from May 2013 until April 2014 among children aged 6 months to 12 years old. A schematic overview of the cohort is presented in Additional file 1. After obtaining written informed consent from a parent or legal guardian, children were clinically examined (including weight, signs of anaemia, symptoms of sickness, permanent disability and chronic illness), and screened for glucose-6-phosphate dehydrogenase (G6PD) deficiency using the BinaxNOW G6PD (Alere Inc., USA) test kit. To assure appropriate test performance, these tests were stored at $4{ }^{\circ} \mathrm{C}$ and conducted on top of an ice bucket, in a well-aerated room $\left(<30{ }^{\circ} \mathrm{C}\right)$. Demographic information (including age, date of birth, sex, residency status, village of residency), LLIN usage, and past treatment of malaria were recorded. Children that fit the inclusion criteria (local healthy individual aged between 6 months and 12 years old with a normal weight that has given consent and completed all enrolment procedures) were invited to participate in the cohort study. Children with a chronic illness or permanent disability, living in a remote area and children that were not permanent residents were excluded. All eligible and consenting children were enrolled.

Participants were then actively followed for a period of 11 months at 4-weekly intervals, apart from a longer period (up to 6 weeks) between the 7th and 8th visit due to the Christmas holiday season. During each active case detection (ACD) visit, information on LLIN usage the night before by mother and child was recorded. The reported LLIN usage by the participants and their mothers was very similar and thus was combined and averaged the LLIN usage data from both mother and child during cohort. Tympanic temperature and antimalarial treatment history (from each child's personal health book) were also recorded.

Any child presenting with fever (temperature $\geq 38{ }^{\circ} \mathrm{C}$ ) or history of fever (recorded up to 2 weeks prior to examination) was clinically assessed by a health worker. If malaria was suspected, a rapid diagnostic test (RDT) (CareStart, Access Bio, USA) was performed. Treatment for malaria was given in accordance with the national malaria treatment guidelines: artemether-lumefantrine $\left(\right.$ Coartem $\left.{ }^{\circledR}\right)$ treatment for P. falciparum infections; Coartem $^{\circledR}$ and 14 days of primaquine (PQ; $0.25 \mathrm{mg} / \mathrm{kg}$, only in G6PD normal individual) for P. vivax infections. Guidelines require G6PD testing prior to PQ treatment. Haemoglobin $(\mathrm{Hb})$ was measured if a child had signs of anaemia (conjunctiva, hand pallor, oedema, shortness of breath or feeling dizzy). Children were treated according to diagnosis and/or, when appropriate, referred to Tulagi Hospital. Participants with frequent absenteeism (less than $6 \mathrm{ACD}$ visit attendances) were excluded from further analysis.

A capillary blood sample (approximately $250 \mu \mathrm{L}$ ) was collected in EDTA-Microtainer (Becton-Dickinson, NJ, USA) tubes from all participants. At the collection site, $50 \mu \mathrm{L}$ of blood were immediately transferred into $250 \mu \mathrm{L}$ RNAProtect (Qiagen, Germany) RNA stabilization reagent, and stored in an ice container (at approximately $4{ }^{\circ} \mathrm{C}$ ) before transportation to the centralized field laboratory. Upon arrival at the centralized field laboratory (within $12 \mathrm{~h}$ of collection), samples in RNA stabilization reagent were frozen immediately at $-20^{\circ} \mathrm{C}$. The remaining blood samples were centrifuged at $300 \times g$ for $10 \mathrm{~min}$ to separate into cellular and plasma fractions, prior to storage at $-20{ }^{\circ} \mathrm{C}$. Blood smears for light microscopy were prepared at ACD1, ACD8 and ACD11, and examined for parasites by experienced microscopists at the regional malaria laboratory. Information from blood smears positive for Plasmodium spp. was used to identify infected children during the survey by the local health workers for anti-malarial treatment. Hb levels were measured at ACD1, ACD6 and ACD11. At the last visit (ACD11), information on participant household indoor residual spraying (IRS) status in the previous 12 months was collected.

A passive case detection (PCD) system was maintained throughout the study at the local health facility levels (Tulagi hospital, local rural health centres and aid posts). Participants identified by PCD were clinically assessed and examined for malaria by RDT. Clinical diagnosis was recorded, and capillary blood samples were collected onto filter paper (Whatman 3MM, Maidstone, UK). Treatment was given to children as per diagnosis of the attending physician.

\section{Molecular characterization of Plasmodium spp. parasites} All collected blood cell pellets (frozen state) and filter paper blood spots (room temperature) were transported to the Walter \& Eliza Hall Institute in Melbourne, 


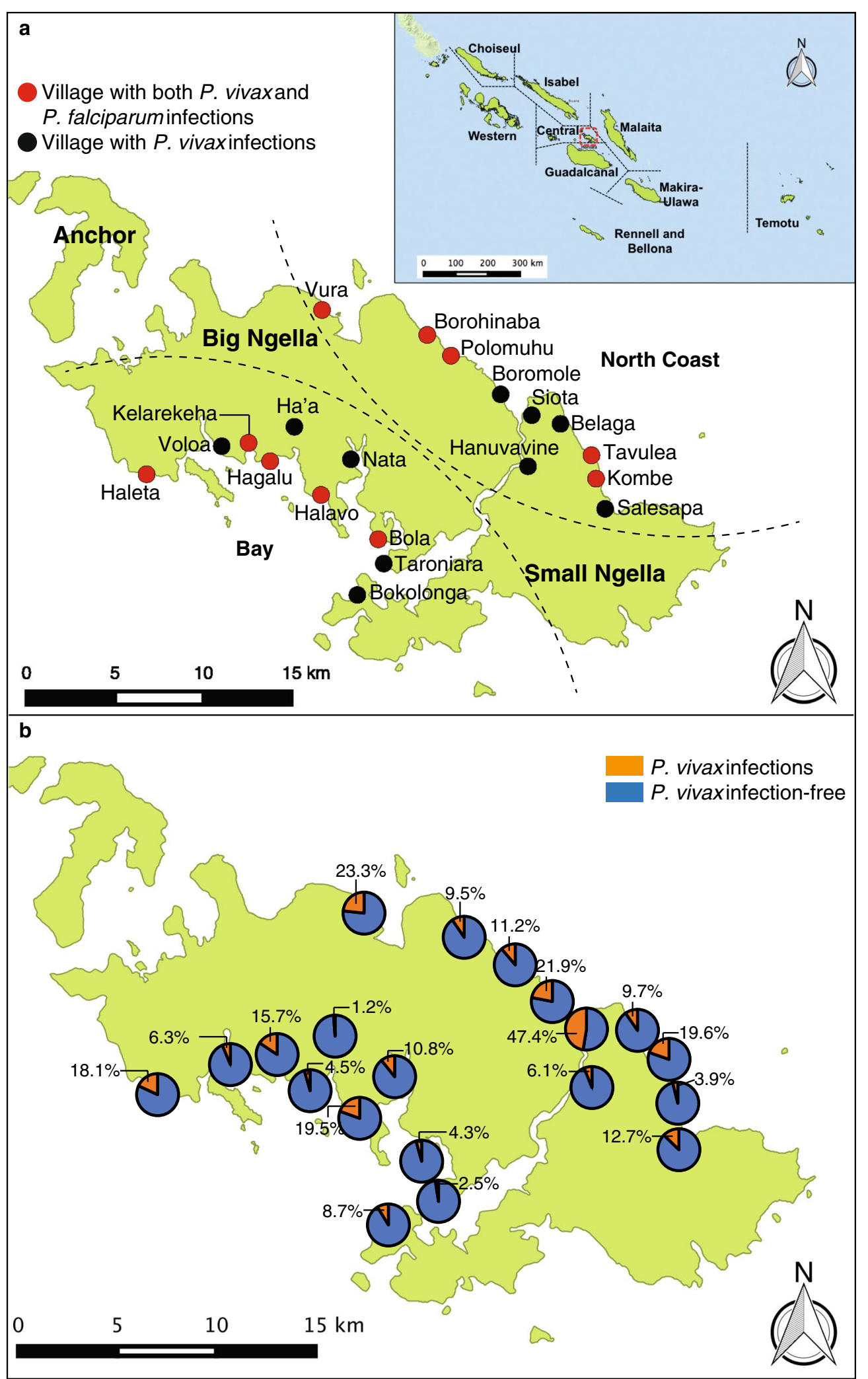

Fig. 1 Ngella study sites and the distribution of Plasmodium spp. infections. a Participants were recruited from 20 villages in Ngella, Central Islands Province. Based on qPCR detection, P. vivax infections were identified in all villages while P. falciparum were detected in 10 villages (red dot). $\mathbf{b}$ Prevalence of $P$. vivax infections (qPCR detection) in Ngella villages (Map created using QGIS 2.10.1) 
Australia. Total genomic DNA was isolated from all blood cellular fractions and filter paper blood spots using FavorPrep 96-Well Genomic DNA Extraction Kits (Favorgen Biotech Corporation, Taiwan). All DNA extracts were screened for $P$. falciparum and $P$. vivax parasites using a duplex $P$. falciparum/P. vivax Taqman qPCR targeting the $18 \mathrm{~s}$ gene [32]. Parasite densities by qPCR are expressed as 18 s gene copy numbers $/ \mu \mathrm{L}$.

Samples positive for parasites were genotyped using molecular markers msp1F3, MS2 and MS16 for P. vivax infections, while $m s p 2$, TA81 and Poly $\alpha$ were used for $P$. falciparum infections [33, 34]. The genotyped samples were submitted for capillary electrophoresis, followed by fragment analysis using GeneMapper software (Applied Biosystems, USA), as described previously [33].

From the genotyping data, the multiplicity of infection (MOI) and molecular force of blood-stage infection ( ${ }_{\text {mol }}$ FOB) were determined $[35,36]$. Briefly, mol $F O B$ is the observed number of new blood-stage infections, as identified by individual Plasmodium spp. genotypes, divided by the time-at-risk (i.e. the incidence of new blood-stage infections). An observed infection, as identified by a specific genotype, was considered to be 'new' if the same genotype (of any molecular marker) had not been seen in the two previous active or passive case detection visits. As such, ${ }_{\text {mol }}$ FOB can be determined for each study participant or as a sum over the entire study population or subpopulations.

\section{Alpha (a)-thalassaemia genotyping}

All children were genotyped for the two most common forms of $\alpha$-thalassaemia: $3.7 \mathrm{kbp}\left(\alpha^{-3.7}\right)$ and the $4.2 \mathrm{kbp}$ deletions $\left(\alpha^{-4.2}\right)$. A multiplex PCR reaction described elsewhere [37] was used and the genotypes were identified by sizing patterns on agarose gel.

\section{Statistical analyses}

Field data were double entered using a REDCap electronic data capture system. All statistical analyses were conducted using STATA (v.12.1, StataCorp) and were focused mainly on $P$. vivax infections as there were too few $P$. falciparum infections to conduct meaningful statistical analysis, except for the analysis of incidence of clinical episodes, where both $P$. vivax and $P$. falciparum data were used. The analyses performed were similar to a previously described paediatric cohort study in Papua New Guinea [38]. Three types of analyses were conducted for $P$. vivax infections: (1) prevalence of infection, (2) incidence of new infection, and (3) infection density. All three analyses were done using a generalized estimating equation (GEE) population-averaged model with the semi-robust Huber/White/sandwich estimator of variance. This approach was taken due to the irregular data structure that included missing data.

The prevalence of $P$. vivax infection (1) throughout the period of the study was analysed using logistic regression based on the XTLOGIT command in STATA. Only the ACD visits data were included in the analysis of prevalence as the study was estimating the prevalence at each visit. The incidence of new $P$. vivax clones acquired during cohort (2) was assessed using a negative binomial regression approach based on the XTNBREG command in STATA [35]. New P. vivax clones acquired during PCD, as determined using the technique described above were binned into the following ACD visit interval for consistency. Plasmodium vivax log-transformed infection densities (3) were analysed by linear regression method using the XTREG command in STATA. Incidences of clinical malaria (both $P$. falciparum and $P$. vivax infections) were analysed using the Andersen and Gill model of multiple time failure survival [39]. Backward elimination using the Wald's Chi square test for individual variables was used to select the best fitting models in all statistical analyses. Associations between risk predictors from models were assessed using Chi square test, Kruskal-Wallis test or one-way analysis of variance (ANOVA), depending on the variable type.

\section{Results}

\section{Study demographics}

A total of 1111 children aged 6 months to 12 years old from 20 villages in Ngella (Fig. 1) were enrolled during May 2013. Of these, data from 860 children was kept for analysis (Fig. 2). Among the 251 excluded children, 28 children were excluded post-enrolment (refer to Fig. 2 for details), 63 children withdrew from the study; and 160 children were excluded post hoc due to inconsistency in follow-up attendance. The excluded participants (Additional file 2) were similar in gender and age to those included, but due to problems with accessibility of villages a significantly higher number of children residing in the North Coast region were excluded as compared to the Bay region ( $p$-value $<0.001$ ). The 860 children included in the final analyses had a minimum of 6 ACD attendances (Additional file 3).

The demographic characteristics of the 860 included children are listed in Additional file 4. These children came from 469 households, had a median age at enrolment of 5.6 years (IQR: $3.0-8.4$ years), and were $48.4 \%$ female and $51.6 \%$ male. Almost all of children (99\%) were local Ngella residents. The number of children enrolled from the 20 villages varied, ranging from 14 (Nata) to 78 (Polomuhu). Each region (Bay and North Coast) was approximately equally represented. Mean usage of LLIN 


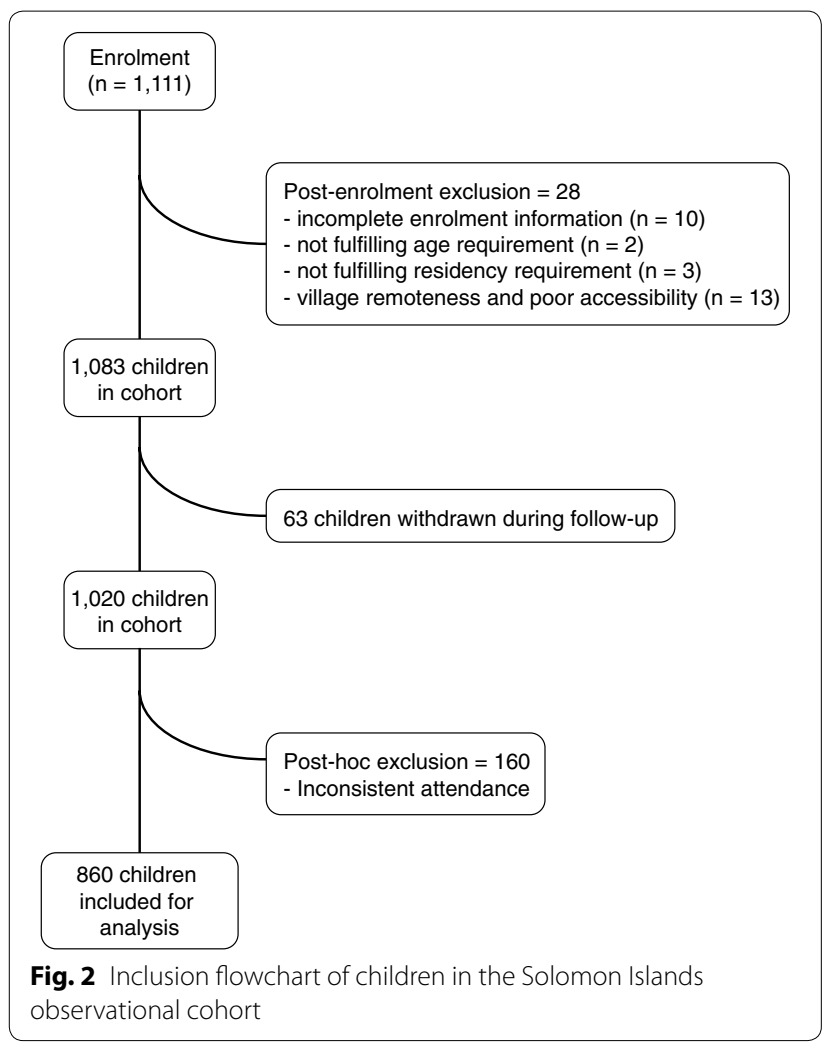

was high at $88 \%$, and $89 \%$ of households were protected by IRS.

During enrolment, $7.2 \%$ of children reported feeling ill. Based on health book records, there were $65.0 \%$ children with at least one previous antimalarial treatment record (PATR), and 35.4\% of children reported having received antimalarial treatment less than 1 year prior to the start this study. Although past parasite species had not been recorded for these previous episodes, it was observed that Coartem ${ }^{\circledR}$ was most commonly given (75.7\%), while PQ treatment was less frequent (20.0\%).

The average $\mathrm{Hb}$ measurements from ACD1 (June 2013), ACD6 (November 2013) and ACD11 (April 2014) showed that $49.8 \%$ of children were anaemic $(\mathrm{Hb}<110 \mathrm{~g} / \mathrm{L})$. There were $10.8 \%$ of children diagnosed as G6PD-deficient, of which $63.4 \%$ were male. Meanwhile, $34.8 \%$ of children had at least one mutated $\alpha$-thalassaemia gene $(89.3 \%$ children with heterozygous polymorphisms and $10.7 \%$ children with homozygous polymorphisms; refer to Additional file 4 ), with the $-\alpha^{3.7}$ being the dominant deletion mutation $(72.8 \%)$.

\section{Burden of Plasmodium spp. infections}

A total of 8329 blood samples were collected and analysed. Based on qPCR screening, 989 P. vivax infections (average prevalence: 11.9\%; 976 observations from ACD visits) and 27 P. falciparum infections (overall prevalence: $0.32 \%$; 26 observations were from ACD visits) were identified, six of which were mixed infections with $P$. vivax. Plasmodium falciparum infections were detected in children from ten villages (Fig. 1). Four of 22 children were positive for $P$. falciparum more than once. These observations indicate that the level of local $P$. falciparum transmission in Ngella is very low. In contrast, ongoing transmission of $P$. vivax infection was observed in Ngella without marked seasonality (Fig. 3). Plasmodium vivax infections showed pronounced clustering among children. Only 294 children (34.2\%) had one or more positive P. vivax blood-stage infections during follow-up (Additional file 5 ). This is significantly fewer than predicted if infections were randomly distributed among children (expected 68.3\%, $\mathrm{p}<0.001$ ). Among those with detected infections, 181 children (21.1\%) had two or more $P$. vivax infections during the follow-up (expected 31.9\%, $\mathrm{p}<0.001$ ) (Additional file 5). Although there was a higher prevalence of G6PD deficiency among male children, the prevalence of $P$. vivax infections was similar among G6PD deficient individuals of both genders $(13.6 \%$ vs. $8.8 \%$ by microscopy or RDT detection, $37.3 \%$ vs. $35.3 \%$ by qPCR detection; Additional file 4).

\section{Genetic diversity, $\mathrm{MOI}$ and ${ }_{\text {mol }} \mathrm{FOB}$}

Of the $P$. vivax positive samples, $90 \%$ were successfully genotyped for msp1F3 alleles, 91\% for both MS2 and MS16 alleles. Meanwhile, only 16 of 27 (59\%) of P. falciparum infected samples were successfully characterized for $m s p 2$ genotypes, but $96 \%$ and $70 \%$ of the P. falciparum samples were successfully genotyped by TA 81 and Polya, respectively (Additional file 6). Based on the calculated virtual heterozygosity $\left(\mathrm{H}_{\mathrm{E}}\right)$ of the first genotyped Plasmodium spp. infection of each infected participant, the $P$. vivax alleles $\left(\mathrm{H}_{\mathrm{E}}\right.$ of $m s p 1 \mathrm{~F} 3=0.78 ; \mathrm{H}_{\mathrm{E}}$ of $\mathrm{MS} 2=0.92 ; \mathrm{H}_{\mathrm{E}}$ of MS16 =0.94; and $\mathrm{H}_{\mathrm{E}}$ of 3 markers combined $=0.98$ ) were found to be more diverse than the $P$. falciparum alleles $\left(\mathrm{H}_{\mathrm{E}}\right.$ of $m s p 2=0.51 ; \mathrm{H}_{\mathrm{E}}$ of TA81 $=0.50 ; \mathrm{H}_{\mathrm{E}}$ of Poly $\alpha=0.24$; and $\mathrm{H}_{\mathrm{E}}$ of TA81 and Poly $\alpha$ markers combined $=0.48)$ (Fig. 4).

In $P$. vivax infections, the MOI ranged from 1 to 3 , with $73 \%$ of infections exhibiting $\mathrm{MOI}=1$. The ${ }_{\text {mol }} \mathrm{FOB}$ was $0.62\left(\mathrm{CI}_{95}\right.$ 0.53-0.72; range 0-14.47) new $P$. vivax infections per child per year-at-risk by msp $1 \mathrm{~F} 3,0.75$ by MS2 $\left(\mathrm{CI}_{95}\right.$ 0.64-0.86; range $\left.0-9.95\right)$ and 0.72 by $\mathrm{MS} 16\left(\mathrm{CI}_{95}\right.$ 0.61-0.83; range 0-12.79). Combining all three molecular markers, the average ${ }_{\text {mol }} \mathrm{FOB}$ was $1.06\left(\mathrm{CI}_{95} 0.90-1.21\right.$, range $0-21.70$ ).

Almost all identified P. falciparum infections exhibited $\mathrm{MOI}=1$, except for three with $\mathrm{MOI}=2$. A proportion of $69 \%$ of the genotyped $P$. falciparum clones shared the same 508 bp msp2 FC27 subtype allele, which was also 

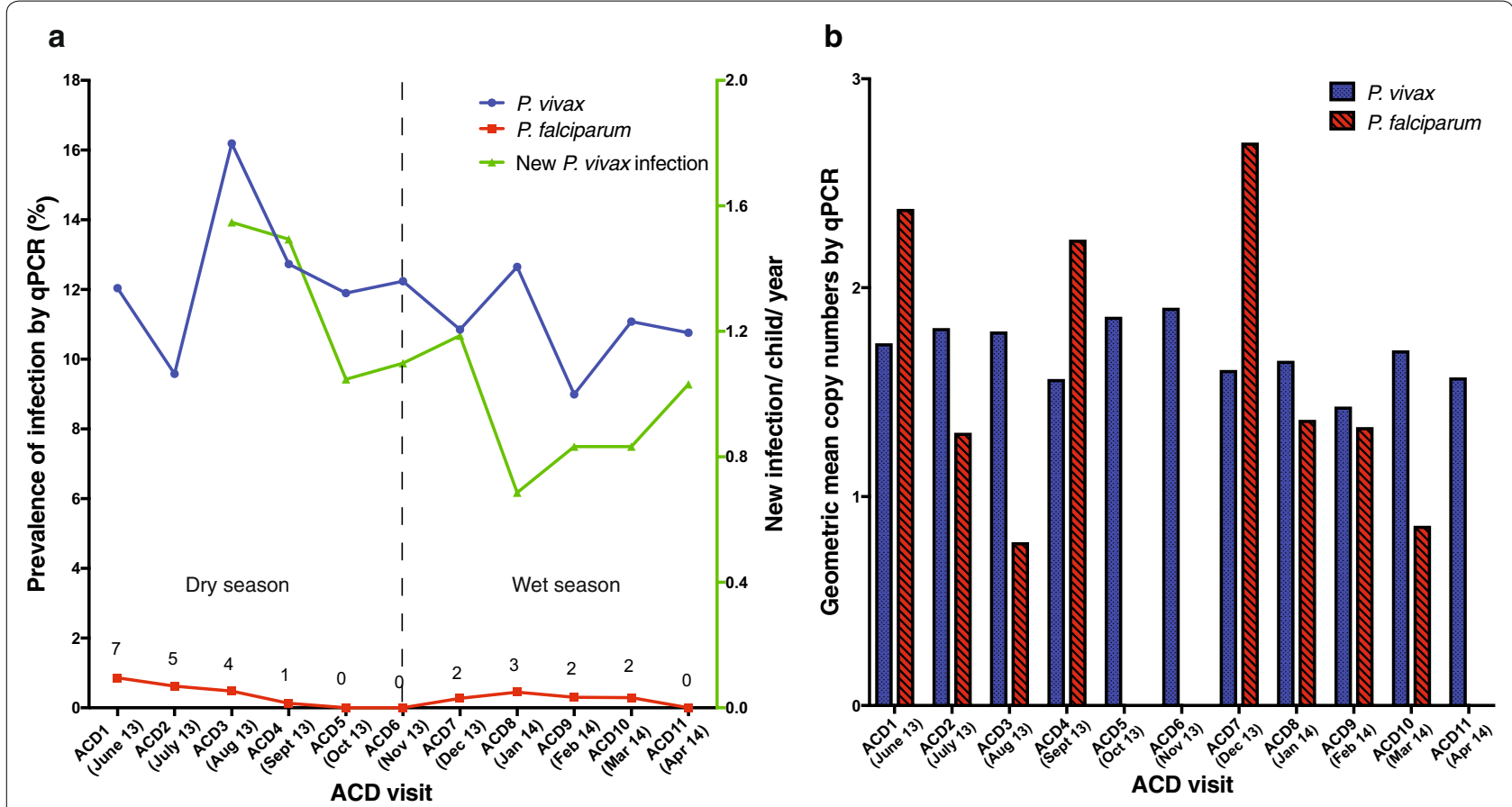

Fig. 3 Plasmodium spp. infections at ACD visits. a Prevalence of qPCR-detected P. falciparum (red curve) and P. vivax (blue curve) infections by monthly ACD follow-up visits (left y-axis). New P. vivax infections (green curve, right $y$-axis) was identified through new genotypes observed in consecutive follow-up blood samples from participants during cohort. $\mathbf{b}$ Infection density of $18 \mathrm{~S}$ rRNA gene of $P$. falciparum and $P$. vivax by qPCR detection

observed previously in the cross-sectional survey during the previous year [23]. For P. falciparum, the average molFOB was $0.02\left(\mathrm{CI}_{95} 0.01-0.04\right.$; range $\left.0-2.91\right)$.

\section{Asymptomatic and submicroscopic Plasmodium spp. infections}

A total of 433 children had at least one febrile episode detected during the active follow-up (ACD) visits. Of these children, 73 were positive by qPCR (71P. vivax and 4 P. falciparum including two mixed infections). As such, an estimated $7.2 \%$ of $P$. vivax and $14.8 \%$ of $P$. falciparum infections were associated with febrile symptoms. All febrile $P$. falciparum and mixed infections but only $46 \%$ of $P$. vivax infections fulfilled the definition of clinical malaria based on febrile assessment and positive parasite detection by RDT or microscopy [40].

Among the 2250 blood films examined during the first (ACD1-June 2013), mid-cohort (ACD8-January 2015) and last visits (ACD11-April 2014), only 78 (3.5\%) blood films were parasite positive by microscopy (76 P. vivax, 4 P. falciparum including two mixed infections) (Additional file 7). Using these data, the proportion of asymptomatic and submicroscopic infections in these 3 follow-up visits were determined: $94 \%$ (247/264) of $P$. vivax and $90 \%(9 / 10)$ of $P$. falciparum infections were asymptomatic, while $71 \%(188 / 264)$ of $P$. vivax and $60 \%$
(6/10) of $P$ falciparum infections were submicroscopic (Additional file 7).

\section{Risk predictors for $P$. vivax infection}

Risk factors for infection prevalence, molFOB and infection density are given in Table 1.

\section{Plasmodium vivax infection prevalence}

Prevalence of $P$. vivax was found to be significantly associated with increased age, G6PD deficiency, LLIN usage, previous antimalarial treatment record (PATR), village of residence and ACD visits (Table 1). Plasmodium vivax prevalence also varied over time $(\mathrm{p}=0.002)$ but did not show a clear seasonal trend (Fig. 3a and Additional file 8). The risk of having a $P$. vivax infection increased linearly with age among children in Ngella (adjusted odds ratio, a OR: $\left.1.18, \mathrm{CI}_{95}[1.13-1.23], \mathrm{p}<0.001\right)$. The risk of $P$. vivax infection varied among villages $(\mathrm{p}<0.001)$ : Compared with Borohinaba, Siota was identified as having the highest $P$. vivax infections risk $\left({ }_{\mathrm{a}} \mathrm{OR}: 6.86, \mathrm{CI}_{95}[2.68-17.5]\right)$ while Ha'a and Taroniara showed the lowest risk of infections ( ${ }_{\mathrm{a}} \mathrm{OR}: 0.18, \mathrm{CI}_{95}[0.022-1.41]$ and ${ }_{\mathrm{a}} \mathrm{OR}: 0.18, \mathrm{CI}_{95}$ [0.050-0.62], respectively). The North Coast region of Ngella had a higher infection risk compared to the Bay region (Additional file 9). Despite this, heterogeneity of infection was observed; children living in Kombe (North 


\section{Diversity of $\boldsymbol{P}$. vivax}

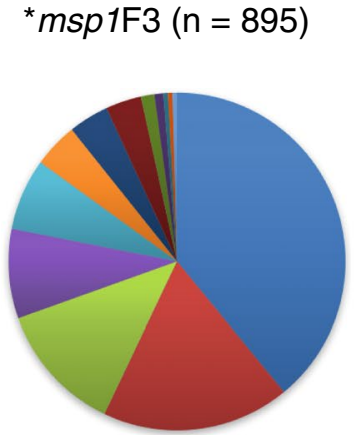

$H_{E}=0.78$
${ }^{*} \mathrm{MS} 2(\mathrm{n}=900)$

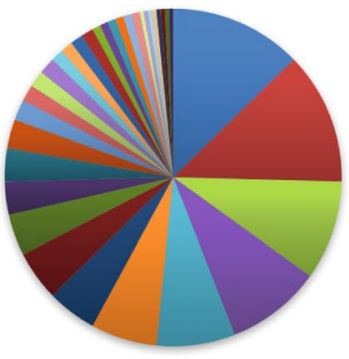

$H_{E}=0.92$

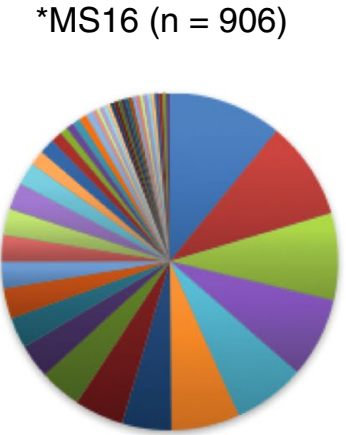

$H_{E}=0.94$

\section{** msp1F3-MS2-MS16} $(n=812)$

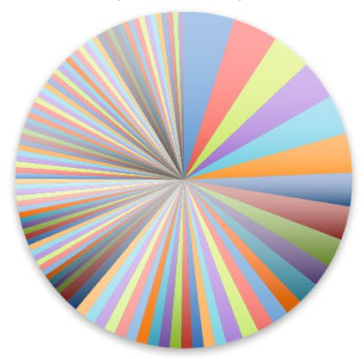

$H_{E}=0.98$

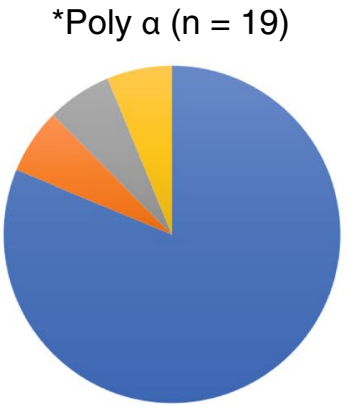

$H_{E}=0.24$

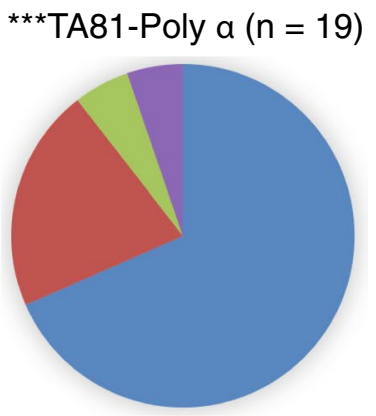

$\mathrm{H}_{\mathrm{E}}=0.48$

Note:

* $\quad$ First $P$. vivax/P. falciparum positive infection

** $\quad$ First $P$. vivax positive infection and $\mathrm{MOI}=1$ for all 3 genotyping markers

$\star \star * \quad$ First $P$. falciparum positive infection and $\mathrm{MOI}=1$ for TA81 and Poly a markers; msp2 marker was excluded as the number of samples that were successfully genotyped were low

Fig. 4 Contrasting diversity of P. vivax and P. falciparum in Ngella. Allelic frequencies of P. vivax markers: msp1F3, MS2, MS16 and the combined msp1F3-MS2-MS16 haplotypes and P. falciparum markers: msp2, TA81, Polya and the combined TA81-Polya haplotypes. The number of successfully genotyped Plasmodium spp. isolates or combined haplotypes are shown above each respective pie chart. Each estimated expected heterozygosity $\left(H_{E}\right)$ are given below the figure

Coast region) had a much lower risk of infection $\left({ }_{\mathrm{a}} \mathrm{OR}\right.$ : $0.66, \mathrm{CI}_{95}$ [0.28-1.59]) while children living in Halavo (Bay region) had a relatively high risk of infection $\left({ }_{\mathrm{a}} \mathrm{OR}\right.$ : $\left.3.15, \mathrm{CI}_{95}[1.50-6.64]\right)$. These differences between villages were stable throughout the cohort study (Additional file 10).

With every $10 \%$ increased of the average LLIN usage, there was an $11 \%$ significant decrease in odds ratio, thus suggesting significant protective effect against $P$. vivax infections by LLIN ( ${ }_{\mathrm{a}} \mathrm{OR}: 0.89, \mathrm{CI}_{95}$ [0.82-0.97], $\mathrm{p}=0.006$ ). Indoor residual spraying (IRS, based on data of 754 participants only) was associated with protection against $P$. vivax infections (OR: $0.42, \mathrm{CI}_{95}$ [0.272-0.650], $\mathrm{p}<0.001$ ) in univariate (Additional file 9A) but not in multivariate analyses.

Children with PATR within 1 year of the start of the cohort study showed a higher risk of $P$. vivax infection ( ${ }_{\mathrm{a}} \mathrm{OR}$ : 2.03, $\mathrm{CI}_{95}$ [1.45-2.83], $\mathrm{p}<0.001$ ). Similarly, G6PD-deficient children were observed to have higher risk of being infected by $P$. vivax ( ${ }_{\mathrm{a}} \mathrm{OR}: 1.77, \mathrm{CI}_{95}[1.16-$ 2.70], $\mathrm{p}=0.008) . \alpha$-Thalassaemia mutation was found to increase (OR: 1.43, $\mathrm{CI}_{95}$ [1.07-1.93], $\mathrm{p}=0.017$ ) the risk of $P$. vivax infections) in univariate analyses only. There were no significant associations between being anaemic $(\mathrm{Hb}<11 \mathrm{~g} / \mathrm{dL})$ and risk of $P$. vivax infections (Additional file $9 \mathrm{~A})$. 


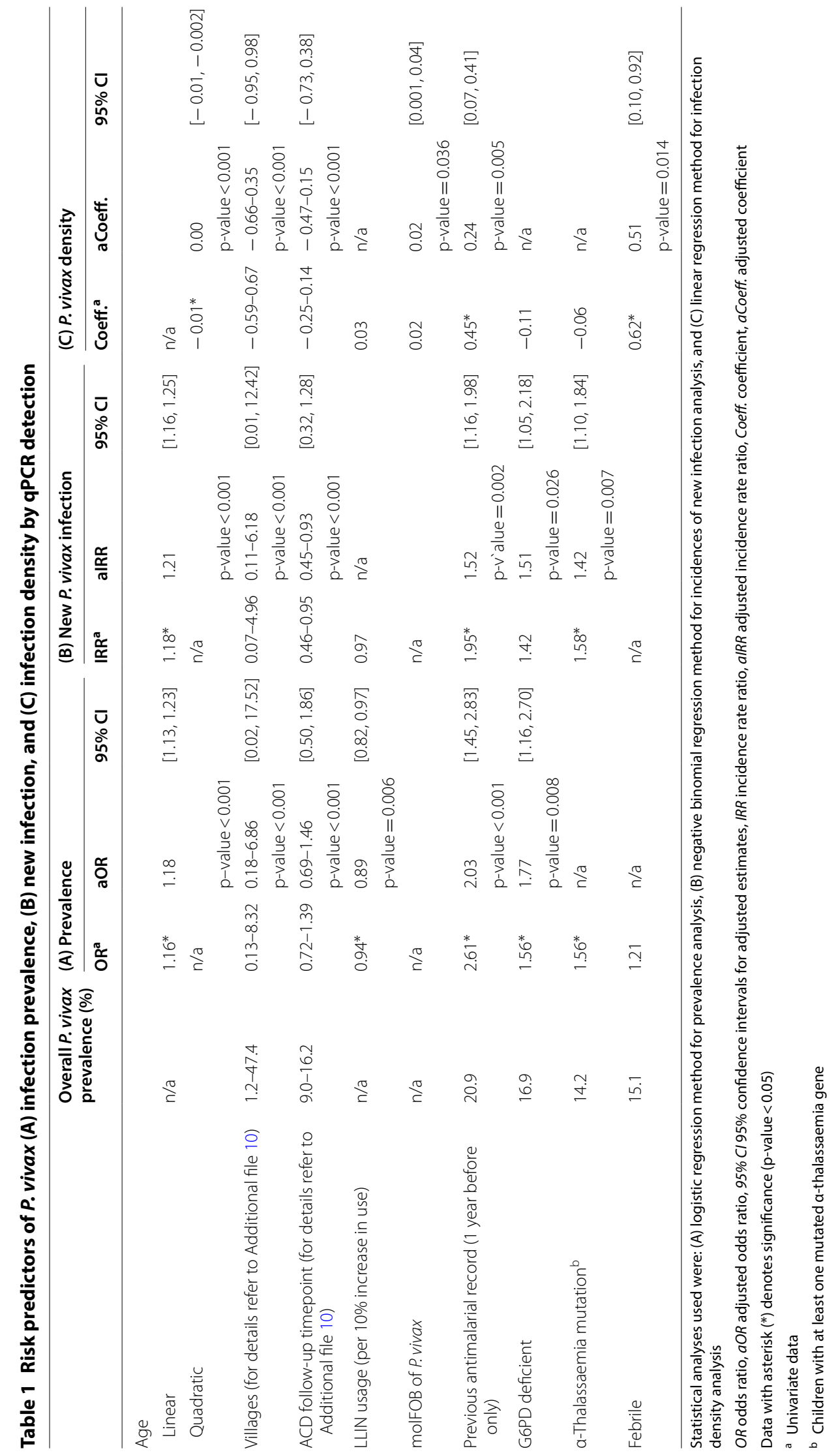




\section{Acquisition of new $P$. vivax blood-stage infection}

Acquisition of new, genetically distinct $P$. vivax bloodstage clones (i.e. mol $\mathrm{FOB}$ ) was also strongly associated with age, villages, visit intervals, PATR, G6PD deficiency and $\alpha$-thalassaemia mutation (Table $1 \mathrm{~b}$ ). As for prevalence, the risk of acquiring new $P$. vivax clones increased linearly with age (adjusted incidence rate ratio, ${ }_{a} I R R$ : 1.21, $\mathrm{CI}_{95}$ [1.16-1.25], $\left.\mathrm{p}<0.001\right)$. Likewise, there was significant heterogeneity in risks among villages $(\mathrm{p}<0.001)$. Risk of new $P$. vivax infections decreased substantially from third visit (reference visit) to last visit ( ${ }_{\mathrm{a}} \mathrm{IRR}$ : 0.61, $\mathrm{CI}_{95}[0.44-0.86]$ ), with fluctuations between visit intervals (Fig. 3a). Children with PATR within 1 year preceding the start of the cohort study ( ${ }_{\mathrm{a}} \mathrm{IRR}: 1.52, \mathrm{CI}_{95}[1.16-1.98]$, $\mathrm{p}=0.002$ ), those with G6PD deficiency ( ${ }_{\mathrm{a}} \mathrm{IRR}: 1.51, \mathrm{CI}_{95}$ [1.05-2.18], $\mathrm{p}=0.026)$ and/or $\alpha$-thalassaemia mutations (IRR: $\left.1.42, \mathrm{CI}_{95}[1.10-1.84], \mathrm{p}=0.007\right)$, were all more likely to acquire new $P$. vivax infections.

LLIN usage did not show any significant association univariately (Additional file 9B) or multivariately with risk of new $P$. vivax infections. Meanwhile, children living in a sprayed household (based on data of 754 participants only; IRR: $0.548, \mathrm{CI}_{95}$ [0.37-0.82], $\mathrm{p}=0.004$ ) were protected from new $P$. vivax infections based on univariate analysis (Additional file 9B). Anaemic children (IRR: 0.696, $\mathrm{CI}_{95}$ [0.52-0.92], $\mathrm{p}=0.012$ ) were observed to have protection against new $P$. vivax infections based on univariate analysis (Additional file 9B). However after adjustment for age, G6PD deficiency, $\alpha$-thalassaemia mutation, PATR, villages and visit intervals, household spraying and anaemic status were no longer significantly associated with incidence of new $P$. vivax infections.

\section{Plasmodium vivax infection density}

Among children with $P$. vivax infections detected during ACD visits $(n=291$; Table $1 c)$, age, PATR, ${ }_{\text {mol }} F O B$, febrile presentation, villages and study visit intervals were significantly associated with the geometric mean density of $P$. vivax infections. The $P$. vivax infection density decreased quadratically ( ${ }_{\mathrm{a}}$ Coefficient: $-0.004, \mathrm{CI}_{95}[-0.006$ to -0.002 ], $\mathrm{p}<0.001$ ) with age and varied significantly among villages $(\mathrm{p}<0.001)$. Contrary to infection risk, a seasonal pattern was observed for $P$. vivax infection density (Fig. 3b) with parasite densities significantly higher during the dry season (ACD1 to ACD5, June-October 2013) as compared to the wet season (ACD6 to ACD11, November 2013-April 2014). Overall, parasitemia decreased significantly $(\mathrm{p}<0.001)$ with increasing time of follow-up.

Children presenting with febrile illness had $P$. vivax infections with higher parasitaemia (Coefficient: 0.51, $\mathrm{CI}_{95}$ [0.10-0.92], $\left.\mathrm{p}=0.014\right)$. Similarly, $P$. vivax infections among children with higher $P$. vivax exposure were also observed to have significantly higher parasite density ( ${ }_{\mathrm{a}}$ Coefficient: $0.02, \mathrm{CI}_{95}$ [0.001-0.04], $\mathrm{p}=0.036$; per unit increase in $\left.{ }_{\text {mol }} \mathrm{FOB}\right)$. Likewise, $P$. vivax infected children with PATR within 1 year prior to the start of cohort had significantly higher parasite densities $\left({ }_{\mathrm{a}}\right.$ Coefficient: 0.24 , $\left.\mathrm{CI}_{95}[0.07-0.41], \mathrm{p}=0.005\right)$.

Reduction of parasitaemia was observed to be significantly associated with IRS univariately (Coefficient: $-0.29, \mathrm{CI}_{95}$ [-0.56 to -0.02$], \mathrm{p}=0.035$; Additional file $9 \mathrm{C}$ ) and multivariately ( ${ }_{\mathrm{a}}$ Coefficient: $0.91, \mathrm{CI}_{95}[-1.37$ to -0.45 ], $\mathrm{p}<0.001$; Additional file 8 ), though this observation is based on only the 256 infected children with IRS information instead of all infected children $(n=291)$. G6PD deficiency, $\alpha$-thalassaemia mutation, mean LLIN usage and anaemia were not correlated with $P$. vivax infection density.

\section{Malaria incidence}

Due to unforeseen staffing and reporting problems, the quality of passive case detection was highly variable during follow-up. To overcome this issue, three distinct definitions of malaria episodes were used: (1) confirmed malaria episode (febrile and concurrent parasitaemia diagnosed by microscopy or RDT), (2) presumptive malaria (missing ACD morbidity and PCD records but documented antimalarial treatment in the child's personal health book), and (3) potential malaria (febrile and positive detection of Plasmodium spp. by qPCR). Since the qPCR TaqMan detection method is a highly sensitive and more accurate as compared to RDT or light microscopy, all malaria species were corrected based on qPCR species typing to reduce the possibility of misinterpreted RDT results at field sites (13 misdiagnosed $P$. vivax infections as $P$. falciparum in the RDT results were identified). In Solomon Islands, only patients with RDT or light microscopy-confirmed malaria receive anti-malarial treatment. However, the infected species is not generally recorded in the patient's health book. The combination of confirmed (Definition 1) and presumptive (Definition 2) malaria is thus most likely to reflect the true overall burden of clinical malaria episodes reliably.

There were 36 confirmed, 97 presumptive, and 39 potential malaria cases. $P$. vivax accounted for most of the clinical infections $(94.6 \%$ in confirmed and $97.4 \%$ in potential malaria cases). The PATR (adjusted hazard ratio, $\left.{ }_{a} \mathrm{HR}: 2.23, \mathrm{CI}_{95}[1.45-3.42], \mathrm{p}<0.001\right)$ and the number of new infections acquired ${ }_{\text {mol }}$ FOB: 2.31 (cube root-transformed), $\mathrm{CI}_{95}$ [1.77-3.02], $\mathrm{p}<0.001$ ) were associated with increased risk of confirmed and presumptive malaria (i.e. definitions 1 and 2), while age $\left({ }_{a} \mathrm{HR}: 0.89\right.$, $\mathrm{CI}_{95}$ [0.83-0.95], $\left.\mathrm{p}=0.001\right)$ decreased the risk. The same association of PATR and ${ }_{\text {mol }} \mathrm{FOB}$ with increased risk were also found for confirmed cases only (Definition 1) and 
all confirmed, presumptive and potential cases (Definition 1-3), whereas the decreased incidence with age was found only for the latter (Additional file 11).

\section{Plasmodium falciparum infections}

Only 27 P. falciparum infections were observed during the cohort. The densities of these infections vary based on qPCR detection, ranging from 5 to 133,000 copy numbers $/ \mu \mathrm{L}$, while the median of parasite densities was 10.5 copy numbers/ $\mu \mathrm{L}$ (IQR: $5-148$ copy numbers $/ \mu \mathrm{L}$ ). Although the occurrence of $P$. falciparum was observed to be sporadic in Ngella, four individuals with repeated $P$. falciparum infections during follow-up visits were residing in the Bay region (two living in Halavo, and one each from Haleta and Bola). Of these four individuals, only one had a febrile episode once. Gametocyte detection by qPCR was not conducted but no P. falciparum gametocytes were detected during the three ACD visits' microscopy examinations.

\section{Discussion}

This study presents the first prospective cohort study focusing on Plasmodium spp. infection dynamics in Solomon Islands. As recognized in earlier cross-sectional studies in Ngella [23], and in the pilot elimination provinces in Temotu [27] and Isabel [22], most Plasmodium spp. infections detected from the cohort were submicroscopic and asymptomatic $P$. vivax infections. This is in line with the general trend of an increasing proportion of submicroscopic Plasmodium spp. infections as malaria transmission decreases [5, 7, 41]. Plasmodium falciparum infections which was previously thought to be nearly eliminated in Ngella [23] remained low in occurrence but persisted across Ngella sporadically.

The incidence of clinical malaria was low among cohort children and when species determination was possible, P. vivax accounted for most cases. This contrasts with the annual national malaria report by the National Vector-borne Disease Control Programme, Ministry of Health and Medical Sciences of Solomon Islands, which reported that approximately half of the malaria incidence in Central Islands was due to P. falciparum (49\% and 47\% of malaria cases in 2013 and 2014, respectively) [21]. Although information retrieval was attempted by checking for clinical malaria records in the health book of all children, it is likely that the true burden of malaria has been underestimated. It is also possible that $P$. falciparum episodes were more common among cases treated at the health facilities as compared to those diagnosed during active case detection. However, it has been previously recognized that the accuracy of clinical malaria case reporting in primary health care facilities in Solomon Islands is often relatively poor [42]. In addition, the results of this study are well in line with the changes observed at a remote region of Malaita, the neighbouring island to Ngella, where in parallel with a $91 \%$ reduction in all malaria cases, the proportion of clinical cases due to P. vivax increased from $33 \%$ in 2008 to $84 \%$ in 2013 [43]. While further studies will be required to determine the relative contribution of $P$. falciparum and $P$. vivax to the burden of clinical malaria in Ngella, data of this study make it clear that the very high burden of asymptomatic, low-density $P$. vivax infections is the key challenge to future malaria elimination in Ngella.

Although $P$. vivax infections were found throughout the study area, a very high degree of spatial heterogeneity in transmission was observed among the study villages. Prevalence varied up to 40-fold (range 1.2-47.4\%) while the ${ }_{\text {mol }}$ FOB varied up to 90-fold (range 0.05-4.57) between villages that were not more than $5-25 \mathrm{~km}$ apart. Such strong variation in malaria transmission levels at small geographical scales has been described before in both moderate to high transmission areas $[44,45]$ and are even more pronounced in low transmission settings [46, 47]. While the exact reasons for the persistence of these pockets of high transmission remain to be elucidated, the finding has important implications for current control and future elimination efforts in Solomon Islands. The high degree of heterogeneity will require spatial-targeting of malaria control and elimination programmes, where the high-risk villages (i.e. Siota) can be both a 'sentinel site' for surveillance as well as a target for enhanced control efforts, which includes active case detection and/or presumptive mass drug treatment $[3,48]$.

Although the burden of $P$. vivax infections varied during follow-up, seasonal patterns, while present, were modest. After a small drop in prevalence from ACD1 to ACD2, which was due to the fact that children with LMpositive infections detected at baseline $(n=52)$ received antimalarial treatments, prevalence and incidence of infections peaked in August (i.e. ACD 3) and then decreased during further follow-up. This coincided with a significant decrease in infection densities during the rainy season (ACD6 to ACD11; November-April), indicating that infection during the rainy season could be due to relapsing rather than newly acquired $P$. vivax infection. According to a previous environmental study in Northern Guadalcanal (west of Ngella), rainfall has a negative effect on Anopheles farauti larvae numbers as heavy rainfall flushes away the breeding soil ridges by the stream [49], thus explaining this somewhat surprising decrease in transmission levels during the rainy season. In addition to these seasonal effects, the overall declining infections could also be a result of the "healthy cohort" effect [50-52], as participation in an intensive cohort provides participants both with better access to healthcare, and 
makes their parents or guardians more aware of the disease.

Despite more than two-thirds of Anopheline mosquitoes in Solomon Islands bites outdoor and early (before $21.00 \mathrm{~h})[26,53]$, LLINs were still associated with a moderate protective effect against $P$. vivax infection prevalence among cohort children. Distribution of LLINs is undertaken every 3 years as the main vector control intervention in Solomon Islands and a 1.5 people/ net ratio/population was reported for 2013 in Central Islands province [21, 54]. High LLIN ownership was also reported in a later nationwide survey (2015) with $85.9 \%$ of households owning at least one LLIN. However, only $56.8 \%$ people reported sleeping under the bed net [21]. The reasons for not using LLINs are complex but increasingly higher usage indicated that the awareness campaigns conducted along with the distribution of LLIN are working. Although a tendency towards a reduction was seen in the univariate analyses, LLIN use was not associated with significant reduction in incidence of new $P$. vivax blood-stage infections. It remains to be confirmed if this is a consistent effect. In Solomon Islands, IRS is considered to be a complementary intervention to LLINs [55] and only high-risk villages are selected for implementation with varying insecticide dosage [21]. Despite incomplete information on IRS among households, children living in sprayed households had significantly fewer P. vivax infections and lower parasite densities. This supports the continued use of IRS not merely as a complementary intervention in high risk areas but also in elimination areas where interruption of Plasmodium spp. transmission is the ultimate goal [56].

Despite relatively low levels of transmission, children in Ngella acquire substantial levels of clinical immunity to $P$. vivax. Although the prevalence of infections, the incidence of new infections, and thus exposure $\left(\mathrm{mol}_{\mathrm{moB}} \mathrm{FOB}\right)$, all increase with age and exposure is a major risk predictor of clinical $P$. vivax malaria and parasite density, the incidence of clinical $P$. vivax episodes itself is not only very low but also decreases with age as does the mean density of $P$. vivax infections. These observed trends are signs of significant natural acquisition of clinical immunity against $P$. vivax. Similarly rapid acquisition of immunity to $P$. vivax was not only observed in a high transmission setting in PNG, where children start acquiring immunity to $P$. vivax in their 2 nd year of life [38] and clinical $P$. vivax illness is virtually absent by age five [57], but also in lower transmission settings [14]. Although acquisition of immunity differs by endemicity settings and the exact rate of acquisition is unclear, the more rapid acquisition of immunity to $P$. vivax is largely due to the higher molFOB $[36,58]$ driven by the combined burden of new and relapsing infections [11].
Having suffered an episode of malaria in the previous 12 months (previous anti-malarial treatment record or PATR) was found to be a strong risk predictor for infection prevalence, infection density, new infection, and malaria incidence. A higher risk of $P$. vivax infection in children with PATR suggests that treatment policy for $P$. vivax malaria in Solomon Island is sub-optimal. Although primaquine radical cure is part of the Solomon Islands' standard treatment guidelines, it is often not prescribed due to the lack of G6PD testing and when prescribed, it is taken by the patient without supervision. A recent analysis of $>60,000$ patients in Indonesian Papua found that unsupervised PQ was associated with only a minimal reduction in the risk of clinical $P$. vivax recurrences in the next 12 months [59]. As relapse infections can account for $80 \%$ of $P$. vivax blood-stage infections $[11,60]$, the high rate of recurrent $P$. vivax infections in children with PATR underlines that PQ treatment was either not given or not successful in removing hypnozoites from the liver, leading to future relapses. This effect is likely to be confounded by significant inter-individual heterogeneity in $P$. vivax infection risk as indicated by the high degree clustering of $P$. vivax infections among individuals. Having had a previous $P$. vivax infection is thus not only an indication that the child may carry further hypnozoites in its liver, but also indicates children with past exposures are significantly more likely to be exposed to new infectious mosquito bites in future. While ${ }_{\text {mol }} \mathrm{FOB}$ is a marker for current exposure to $P$. vivax infection, PATR is thus also a marker of (recent) past exposure.

The important contribution of relapses to the burden of $P$. vivax is further highlighted by the observation that G6PD deficient children had a higher prevalence of $P$. vivax infection and increased risk of acquiring new infection. This observation conflicts with previous reports that G6PD deficiency confers protection against severe $P$. falciparum malaria and clinical $P$. vivax infections and P. vivax parasitaemia [61-63]. While G6PD deficiency may protect against high $P$. vivax parasitaemia and thus clinical disease, the higher risk among G6PD deficient children is more likely due to the fact that these children represented a group that could not be treated with $P Q$, and therefore only received blood stage treatment. This again underlines the importance of providing efficient anti-relapse treatment and the need to find alternative treatment or prevention strategies for G6PD-deficient individuals.

Observation of low P. falciparum prevalence that are sporadic and largely asymptomatic occurring throughout the study areas and follow-up period is intriguing. Even more so as the National Vector-borne Disease Control Programme [21] reports that half of all clinical cases in Ngella are due to P. falciparum (see 
"Discussion" above). The observed $P$. falciparum in this cohort show low levels of genetic diversity and a large proportion (69\%) carry the same msp2 FC27 allele as the five $P$. falciparum infections detected in the previous year's cross-sectional survey [23]. Likewise, there were low genetic diversity based on TA81 and Poly $\alpha$ characterization. Together, these observations suggest that endemic transmission of a near-clonal $P$. falciparum populations may persist in Ngella, similar to that previously observed in elimination province Temotu [64]. Further in-depth analyses of P. falciparum population genetics in Ngella and comparisons to neighbouring populations, as well as a detailed assessment of clinical isolates compared to asymptomatic infections will be required to better understand the nature of residual $P$. falciparum transmission in Ngella. In particular, it will be essential to understand what proportion and what infections are imported from neighbouring islands, which have much higher endemicity.

\section{Conclusions}

This study has confirmed the presence of a significant burden of $P$. vivax infection and the sporadic occurrence of $P$. falciparum in Ngella, largely asymptomatic and submicroscopic. The Solomon Islands malaria control programme currently relies almost entirely on vector control measures (such as LLINs and IRS) and management of clinical cases. While these approaches which have jointly achieved an overall 95\% reduction in malaria cases in the last two decades remain effective, they are unlikely to achieve elimination of malaria transmission in Solomon Islands on their own. Alternative approaches that are better able to identify and target these silent reservoir Plasmodium spp. infections will be required. Higher sensitivity diagnosis tools such as loop-mediated isothermal amplification (LAMP) or PCR may be required to track these infections, and using active surveillance method rather than relying solely on passive surveillance method may be required to aid malaria elimination in Solomon Islands [65-67]. While active case finding and reactive case detection $[48,68,69]$ may help with eliminating residual foci of $P$. falciparum transmission, further progress with eliminating $P$. vivax will crucially depend on attacking the hidden, hypnozoite reservoir [11]. A first priority would be the implementation of safe and efficacious PQ treatment (including G6PD testing) for all $P$. vivax infected individuals [70]. Further concerted efforts combining accurate identification of pockets of residual high transmission with focal mass drug administration may be required to detect and eliminate the reservoir of asymptomatic and submicroscopic $P$. vivax infections.

\section{Additional files}

Additional file 1: Figure S1. Schematic overview of the paediatric cohort study in Ngella, Solomon Islands.

Additional file 2: Table S1. Characteristics of children that met inclusion criteria vs excluded children.

Additional file 3: Table S2. Retention of cohort participants and malaria prevalence during active case detection.

Additional file 4: Table S3. Detailed demographic characteristics of 860 children included in the cohort study.

Additional file 5: Figure S3. Frequency of Plasmodium vivax infections among cohort participants ( $\mathrm{n}=860$ ).

Additional file 6: Figure S4. Plasmodium falciparum genotypes variants that were successfully characterized by msp2, TA81 and Polya markers.

Additional file 7: Figure S5. Asymptomatic and submicroscopic Plasmodium spp. infections during ACD1, ACD8 and ACD11 visits.

Additional file 8: Table S5. (i) Association between selected risk factors and Plasmodium vivax infection density by qPCR detection of the 256 participants with at least 1 P. vivax infection during cohort and household spraying data. (ii) Detailed estimates for village and visit interval effects for (A) Plasmodium vivax infection prevalence, (B) new P. vivax infection, and (C) P. vivax infection density by $\mathrm{qPCR}$ detection.

Additional file 9: Table S4. Univariate analysis of risk factors associated with (A) Plasmodium vivax infection, (B) new P. vivax infection and (C) P. vivax density by qPCR detection.

Additional file 10: Figure S2. Prevalence of Plasmodium vivax infection in villages during ACD visits.

Additional file 11: Table S6. Risk factors for incidence of clinical episodes: (A) Confirm malaria, presumptive malaria and potential malaria, (B) Confirm malaria.

\section{Authors' contributions}

$\mathrm{IM}$ and JK conceived and designed the study. AW, $\mathrm{V}$ and FP performed the field work. YWQ performed the laboratory work. YWQ, DJT and AW performed data cleaning. YWQ, IM, SK, MTW and DJT analysed the data. YWQ and IM interpreted the data. YWQ wrote the first draft of the manuscript. IM, SK, MTW, MW, DJT, AB, CB and JK provided critical revision of the manuscript for important intellectual content. All authors read and approved the final manuscript.

\section{Author details}

${ }_{1}^{1}$ Population Health and Immunity Division, The Walter and Eliza Hall Institute of Medical Research, Parkville, VIC, Australia. ${ }^{2}$ Department of Medical Biology, The University of Melbourne, Parkville, VIC, Australia. ${ }^{3}$ Department of Parasites and Insect Vectors, Institut Pasteur, Paris, France. ${ }^{4}$ National Health Training \& Research Institute, Ministry of Health, Honiara, Solomon Islands. ${ }^{5}$ College of Public Health, Medical and Veterinary Sciences, James Cook University, Townsville, QLD, Australia. ${ }^{6}$ Case Western Reserve University, Cleveland, OH, USA.

\section{Acknowledgements}

We would like to thank the study participants, their parents and guardians, communities, community leaders for their support and participation in this study. We also thank the field teams, field laboratory, clinics, Tulagi hospital, National Vector-borne Disease Control Programme, Ministry of Health and Medical Sciences of Solomon Islands for their great support in this study.

\section{Competing interests}

The authors declare that they have no competing interests.

\section{Availability of data and materials}

All data generated or analysed during this study are included in this published article and its additional information files. 


\section{Ethics approval and consent to participate}

This study was approved by the Solomon Islands National Health and Ethics Committee (IRB number: HRC12/022), the Walter and Eliza Hall Institute of Medical Research Human Research Ethics Committee (HREC number: 13/03) and the Cleveland Hospital Institutional Review Board (UHCMC IRB number: 06-12-16). Informed written consent was obtained from the parents or guardians of all children prior to enrolment.

\section{Funding}

YWQ was supported by both Melbourne International Fee Remission Scholarship (MIFRS) and Melbourne International Research Scholarship (MIRS), SK is supported by a NHRMC Early Career Fellowship (GNT1052760), IM was supported by an NHMRC Senior Research Fellowship (GNT1043345). This work was supported by National Institutes of Health (1 U19 Al089686-01 and 1 U19 Al129392-01), the National Health \& Medical Research Council (GNT1003825, GNT1102297 and GNT1092789), the Transmission Epidemiology Consortium (supported by the Bill and Melinda Gates Foundation) and the Victorian State Government Operational Infrastructure Support.

\section{Publisher's Note}

Springer Nature remains neutral with regard to jurisdictional claims in published maps and institutional affiliations.

Received: 24 September 2018 Accepted: 14 March 2019

Published online: 28 March 2019

\section{References}

1. WHO. World malaria report 2016. Geneva: World Health Organization; 2016.

2. Bejon P, Williams TN, Nyundo C, Hay SI, Benz D, Gething PW, et al. A microepidemiological analysis of febrile malaria in Coastal Kenya showing hotspots within hotspots. Elife. 2014;3:e02130.

3. Bousema T, Griffin JT, Sauerwein RW, Smith DL, Churcher TS, Takken W, et al. Hitting hotspots: spatial targeting of malaria for control and elimination. PLoS Med. 2012;9:e1001165.

4. Sturrock HJ, Bennett AF, Midekisa A, Gosling RD, Gething PW, Greenhouse B. Mapping malaria risk in low transmission settings: challenges and opportunities. Trends Parasitol. 2016;32:635-45.

5. Cheng Q, Cunningham J, Gatton ML. Systematic review of sub-microscopic P. vivax infections: prevalence and determining factors. PLoS Negl Trop Dis. 2015;9:e3413.

6. Cotter C, Sturrock HJ, Hsiang MS, Liu J, Phillips AA, Hwang J, et al. The changing epidemiology of malaria elimination: new strategies for new challenges. Lancet. 2013:382:900-11.

7. Chen I, Clarke SE, Gosling R, Hamainza B, Killeen G, Magill A, et al. "Asymptomatic" malaria: a chronic and debilitating infection that should be treated. PLoS Med. 2016;13:e1001942.

8. Bousema T, Drakeley C. Epidemiology and infectivity of Plasmodium falciparum and Plasmodium vivax gametocytes in relation to malaria control and elimination. Clin Microbiol Rev. 2011;24:377-410.

9. Howes RE, Battle KE, Mendis KN, Smith DL, Cibulskis RE, Baird JK, et al. Global epidemiology of Plasmodium vivax. Am J Trop Med Hyg. 2016;95(6 Suppl):15-34.

10. Felger I, Maire M, Bretscher MT, Falk N, Tiaden A, Sama W, et al. The dynamics of natural Plasmodium falciparum infections. PLOS ONE. 2012;7:e45542.

11. Robinson LJ, Wampfler R, Betuela I, Karl S, White MT, Suen CSLW, et al. Strategies for understanding and reducing the Plasmodium vivax and Plasmodium ovale hypnozoite reservoir in Papua New Guinean children: a randomised placebo-controlled trial and mathematical model. PLoS Med. 2015;12:e1001891.

12. Feachem RG, Phillips AA, Hwang J, Cotter C, Wielgosz B, Greenwood $\mathrm{BM}$, et al. Shrinking the malaria map: progress and prospects. Lancet. 2010;376:1566-78.

13. Mueller I, Galinski MR, Baird JK, Carlton JM, Kochar DK, Alonso PL, et al. Key gaps in the knowledge of Plasmodium vivax, a neglected human malaria parasite. Lancet Infect Dis. 2009;9:555-66.
14. Mueller I, Galinski MR, Tsuboi T, Arevalo-Herrera M, Collins WE, King CL. Natural acquisition of immunity to Plasmodium vivax: epidemiological observations and potential targets. Adv Parasitol. 2013;81:77-131.

15. Genton B, D'Acremont V, Rare L, Baea K, Reeder JC, Alpers MP, et al. Plasmodium vivax and mixed infections are associated with severe malaria in children: a prospective cohort study from Papua New Guinea. PLoS Med. 2008;5:e127.

16. Maitland K, Williams TN, Bennett S, Newbold CI, Peto TE, Viji J, et al. The interaction between Plasmodium falciparum and P. vivax in children on Espiritu Santo island, Vanuatu. Trans R Soc Trop Med Hyg. 1996;90:614-20.

17. Phimpraphi W, Paul RE, Yimsamran S, Puangsa-art S, Thanyavanich N, Maneeboonyang W, et al. Longitudinal study of Plasmodium falciparum and Plasmodium vivax in a Karen population in Thailand. Malar J. 2008;7:99.

18. Kepha S, Nikolay B, Nuwaha F, Mwandawiro CS, Nankabirwa J, Ndibazza J, et al. Plasmodium falciparum parasitaemia and clinical malaria among school children living in a high transmission setting in western Kenya. Malar J. 2016;15:157.

19. Zhou G, Afrane YA, Malla S, Githeko AK, Yan G. Active case surveillance, passive case surveillance and asymptomatic malaria parasite screening illustrate different age distribution, spatial clustering and seasonality in western Kenya. Malar J. 2015;14:41.

20. Asia Pacific Leaders Malaria Alliance. APLMA Strategic Plan, Roadmap Phase 1: 2016-2020. 2016.

21. National Vector-borne Disease Control Program, Solomon Islands. Annual Malaria Report 2015. 2016.

22. Atkinson JA, Johnson ML, Wijesinghe R, Bobogare A, Losi L, O'Sullivan M, et al. Operational research to inform a sub-national surveillance intervention for malaria elimination in Solomon Islands. Malar J. 2012;11:101.

23. Waltmann A, Darcy AW, Harris I, Koepfli C, Lodo J, Vahi V, et al. High rates of asymptomatic, sub-microscopic Plasmodium vivax infection and disappearing Plasmodium falciparum malaria in an area of low transmission in Solomon Islands. PLoS Negl Trop Dis. 2015;9:e0003758.

24. National Vector-borne Disease Control Program, Solomon Islands. Annual Malaria Report 2016. 2017.

25. WHO. World malaria report 2017. Geneva: World Health Organization; 2017.

26. Russell TL, Beebe NW, Bugoro H, Apairamo A, Collins FH, Cooper RD, et al. Anopheles farauti is a homogeneous population that blood feeds early and outdoors in the Solomon Islands. Malar J. 2016;15:151.

27. Harris I, Sharrock WW, Bain LM, Gray KA, Bobogare A, Boaz L, et al. A large proportion of asymptomatic Plasmodium infections with low and sub-microscopic parasite densities in the low transmission setting of Temotu Province, Solomon Islands: challenges for malaria diagnostics in an elimination setting. Malar J. 2010;9:254.

28. Kelly GC, Hale E, Donald W, Batarii W, Bugoro H, Nausien J, et al. A highresolution geospatial surveillance-response system for malaria elimination in Solomon Islands and Vanuatu. Malar J. 2013;12:108.

29. Kelly GC, Hii J, Batarii W, Donald W, Hale E, Nausien J, et al. Modern geographical reconnaissance of target populations in malaria elimination zones. Malar J. 2010;9:289.

30. National Vector-borne Disease Control Program, Solomon Islands. Annual Malaria Report 2012. 2013.

31. National Vector-borne Disease Control Program, Solomon Islands. Annual Malaria Report 2013. 2014.

32. Rosanas-Urgell A, Mueller D, Betuela I, Barnadas C, Iga J, Zimmerman PA, et al. Comparison of diagnostic methods for the detection and quantification of the four sympatric Plasmodium species in field samples from Papua New Guinea. Malar J. 2010;9:361.

33. Koepfli C, Ross A, Kiniboro B, Smith TA, Zimmerman PA, Siba P, et al. Multiplicity and diversity of Plasmodium vivax infections in a highly endemic region in Papua New Guinea. PLoS Negl Trop Dis. 2011;5:e1424.

34. Anderson TJ, Su XZ, Bockarie M, Lagog M, Day KP. Twelve microsatellite markers for characterization of Plasmodium falciparum from finger-prick blood samples. Parasitology. 1999;119:113-25.

35. Hofmann NE, Karl S, Wampfler R, Kiniboro B, Teliki A, Iga J, et al. The complex relationship of exposure to new Plasmodium infections and incidence of clinical malaria in Papua New Guinea. Elife. 2017;6:e23708.

36. Koepfli C, Colborn KL, Kiniboro B, Lin E, Speed TP, Siba PM, et al. A high force of Plasmodium vivax blood-stage infection drives the rapid 
acquisition of immunity in Papua New Guinean children. PLoS NegI Trop Dis. 2013;7:e2403.

37. Chong SS, Boehm CD, Higgs DR, Cutting GR. Single-tube multiplex-PCR screen for common deletional determinants of a-thalassemia. Blood. 2000;95:360-2.

38. Lin E, Kiniboro B, Gray L, Dobbie S, Robinson L, Laumaea A, et al. Differential patterns of infection and disease with $P$. falciparum and $P$. vivax in young Papua New Guinean children. PLoS ONE. 2010;5:e9047.

39. Andersen PK, Gill RD. Cox's regression model for counting processes: a large sample study. Ann Stat. 1982;10(4):1100-20.

40. WHO. WHO malaria terminology. Geneva: World Health Organization; 2017.

41. Okell LC, Bousema T, Griffin JT, Ouedraogo AL, Ghani AC, Drakeley CJ. Factors determining the occurrence of submicroscopic malaria infections and their relevance for control. Nat Commun. 2012;3:1237.

42. Kunimitsu $A$. The accuracy of clinical malaria case reporting at primary health care facilities in Honiara, Solomon Islands. Malar J. 2009;8:80.

43. Oloifana-Polosovai H, Gwala J, Harrington H, Massey PD, Ribeyro E, Flores $A$, et al. A marked decline in the incidence of malaria in a remote region of Malaita, Solomon Islands, 2008 to 2013. Western Pac Surveill Response J. 2014;5:30-9.

44. Parker BS, Paredes Olortegui M, Penataro Yori P, Escobedo K, Florin D, Rengifo Pinedo $S$, et al. Hyperendemic malaria transmission in areas of occupation-related travel in the Peruvian Amazon. Malar J. 2013;12:178.

45. Carrasco-Escobar G, Gamboa D, Castro MC, Bangdiwala SI, Rodriguez H, Contreras-Mancilla J, et al. Micro-epidemiology and spatial heterogeneity of P. vivax parasitaemia in riverine communities of the Peruvian Amazon: a multilevel analysis. Sci Rep. 2017;7:8082.

46. Parker DM, Matthews SA, Yan G, Zhou G, Lee MC, Sirichaisinthop J, et al. Microgeography and molecular epidemiology of malaria at the Thailand-Myanmar border in the malaria pre-elimination phase. Malar J. 2015;14:198.

47. Mogeni P, Omedo I, Nyundo C, Kamau A, Noor A, Bejon P, et al. Effect of transmission intensity on hotspots and micro-epidemiology of malaria in sub-Saharan Africa. BMC Med. 2017;15:121.

48. Sturrock HJ, Hsiang MS, Cohen JM, Smith DL, Greenhouse B, Bousema $T$, et al. Targeting asymptomatic malaria infections: active surveillance in control and elimination. PLoS Med. 2013;10:e1001467.

49. Bugoro H, Hii J, Russell TL, Cooper RD, Chan BK, Iro'ofa C, et al. Influence of environmental factors on the abundance of Anopheles farauti larvae in large brackish water streams in Northern Guadalcanal, Solomon Islands. Malar J. 2011;10:262.

50. Sutcliffe CG, Kobayashi T, Hamapumbu H, Shields T, Mharakurwa S, Thuma PE, et al. Reduced risk of malaria parasitemia following household screening and treatment: a cross-sectional and longitudinal cohort study. PLoS ONE. 2012;7:e31396.

51. O'Sullivan M, Kenilorea G, Yamaguchi Y, Bobogare A, Losi L, Atkinson JA, et al. Malaria elimination in Isabel Province, Solomon Islands: establishing a surveillance-response system to prevent introduction and reintroduction of malaria. Malar J. 2011;10:235.

52. Lim S, Yasuoka J, Poudel KC, Ly P, Nguon C, Jimba M. Promoting community knowledge and action for malaria control in rural Cambodia: potential contributions of Village Malaria Workers. BMC Res Notes. 2012;5:405.

53. Russell TL, Beebe NW, Bugoro H, Apairamo A, Chow WK, Cooper RD, et al. Frequent blood feeding enables insecticide-treated nets to reduce transmission by mosquitoes that bite predominately outdoors. Malar J. 2016;15:156.

54. National Vector-borne Disease Control Program, Solomon Islands. Annual Malaria Report 2014. 2015.
55. Over M, Bakote'e B, Velayudhan R, Wilikai P, Graves PM. Impregnated nets or DDT residual spraying? Field effectiveness of malaria prevention techniques in solomon islands, 1993-1999. Am J Trop Med Hyg. 2004;71(2 Suppl):214-23.

56. Katureebe A, Zinszer K, Arinaitwe E, Rek J, Kakande E, Charland K, et al. Measures of malaria burden after long-lasting insecticidal net distribution and indoor residual spraying at three sites in Uganda: a prospective observational study. PLoS Med. 2016;13:e1002167.

57. Michon P, Cole-Tobian JL, Dabod E, Schoepflin S, Igu J, Susapu M, et al. The risk of malarial infections and disease in Papua New Guinean children. Am J Trop Med Hyg. 2007;76:997-1008.

58. Mueller I, Schoepflin S, Smith TA, Benton KL, Bretscher MT, Lin E, et al. Force of infection is key to understanding the epidemiology of Plasmodium falciparum malaria in Papua New Guinean children. Proc Natl Acad Sci USA. 2012;109:10030-5.

59. Douglas NM, Poespoprodjo JR, Patriani D, Malloy MJ, Kenangalem E, Sugiarto $P$, et al. Unsupervised primaquine for the treatment of Plasmodium vivax malaria relapses in southern Papua: a hospital-based cohort study. PLoS Med. 2017;14:e1002379.

60. Betuela I, Rosanas-Urgell A, Kiniboro B, Stanisic DI, Samol L, de Lazzari $E$, et al. Relapses contribute significantly to the risk of Plasmodium vivax infection and disease in Papua New Guinean children 1-5 years of age. J Infect Dis. 2012;206:1771-80.

61. Howes RE, Battle KE, Satyagraha AW, Baird JK, Hay SI. G6PD deficiency: global distribution, genetic variants and primaquine therapy. Adv Parasitol. 2013;81:133-201.

62. Louicharoen C, Patin E, Paul R, Nuchprayoon I, Witoonpanich B, Peerapittayamongkol C, et al. Positively selected G6PD-Mahidol mutation reduces Plasmodium vivax density in Southeast Asians. Science. 2009;326:1546-9.

63. Leslie T, Briceno M, Mayan I, Mohammed N, Klinkenberg E, Sibley CH, et al. The impact of phenotypic and genotypic G6PD deficiency on risk of Plasmodium vivax infection: a case-control study amongst Afghan refugees in Pakistan. PLoS Med. 2010;7:e1000283.

64. Gray KA, Dowd S, Bain L, Bobogare A, Wini L, Shanks GD, et al. Population genetics of Plasmodium falciparum and Plasmodium vivax and asymptomatic malaria in Temotu Province, Solomon Islands. Malar J. 2013;12:429.

65. Sattabongkot J, Tsuboi T, Han ET, Bantuchai S, Buates S. Loop-mediated isothermal amplification assay for rapid diagnosis of malaria infections in an area of endemicity in Thailand. J Clin Microbiol. 2014;52:1471-7.

66. Cook J, Aydin-Schmidt B, Gonzalez IJ, Bell D, Edlund E, Nassor MH, et al. Loop-mediated isothermal amplification (LAMP) for point-of-care detection of asymptomatic low-density malaria parasite carriers in Zanzibar. Malar J. 2015;14:43.

67. Bousema T, Okell L, Felger I, Drakeley C. Asymptomatic malaria infections: detectability, transmissibility and public health relevance. Nat Rev Microbiol. 2014;12:833-40.

68. Molina Gomez K, Caicedo MA, Gaitan A, Herrera-Varela M, Arce MI, Vallejo AF, et al. Characterizing the malaria rural-to-urban transmission interface: the importance of reactive case detection. PLoS Negl Trop Dis. 2017;11:e0005780.

69. Cotter C, Sudathip P, Herdiana H, Cao Y, Liu Y, Luo A, et al. Piloting a programme tool to evaluate malaria case investigation and reactive case detection activities: results from 3 settings in the Asia Pacific. Malar J. 2017;16:347.

70. Baird JK, Valecha N, Duparc S, White NJ, Price RN. Diagnosis and treatment of Plasmodium vivax malaria. Am J Trop Med Hyg. 2016;95(6 Suppl):35-51. 